\title{
Comportement physico-chimique des ammoniacates de calcium. Application à la réfrigération solaire
}

\author{
J. Flechon, K. Benhammou. F. Machizaud et G. Godmel \\ Laboratoire de Physique des Dépôts Métalliques, Université de Nancy I, Faculté des Sciences, BP 239, \\ 54506 Vandouvre-lès-Nancy Cedex, France
}

(Reçu le 12 octobre 1984, révisé le 21 juin 1985, accepté le 16 juillet 1985)

\begin{abstract}
Résumé. - Afin d'utiliser dans les meilleures conditions le couple chlorure de calcium-ammoniac en vue de la réfrigération solaire ou du stockage d'énergie, nous examinons l'effet néfaste de l'eau sur ce binaire. La loi donnant la variation du gonflement que subit le sel en absorbant l'ammoniac jusqu'à saturation complète est indiquée. Nous en déduisons qu'il faut prévoir pour le générateur en volume libre de 4 litres environ pour chaque kilogramme de sel pur. Enfin, nous précisons les vitesses d'absorption et de désorption de $\mathrm{NH}_{3}$. Cette étude montre que plus on se rapproche de l'octoammoniacate, plus la vitesse de formation des complexes est faible.
\end{abstract}

\begin{abstract}
In order to use the couple calcium chloride-ammonia in the best conditions for the solar refrigeration or energy stockage, we show the bad effect of the water on the binary. The law giving the variation of the swelling of the salt absorbing ammonia till complete saturation is indicated. We deduce from this that we have to foresee for the generator a free volume of about 4 liters per kilo of pure salt. Finally we specify the absorption and desorption rates of ammonia. This study shows that the closer we are to the neighbourhood of the octoammoniacate, the lower is the complex formation rate.
\end{abstract}

\section{Introduction.}

L'étude bibliographique $[1,2]$ montre que le chlorure de calcium pur forme 4 complexes différents en réagissant avec l'ammoniac sec. Les réactions réversibles sont les suivantes :

$$
\begin{array}{lll}
\mathrm{CaCl}_{2(\mathrm{~s})}+\mathrm{NH}_{3(\mathrm{~g})} \rightleftharpoons \mathrm{CaCl}_{2}\left(\mathrm{NH}_{3}\right)_{(\mathrm{s})} & \text { I } \\
\mathrm{CaCl}_{2}\left(\mathrm{NH}_{3}\right)_{(\mathrm{s})}+\mathrm{NH}_{3(\mathrm{~g})} \rightleftharpoons \mathrm{CaCl}_{2}\left(\mathrm{NH}_{3}\right)_{2(\mathrm{~s})} & \text { II } \\
\mathrm{CaCl}_{2}\left(\mathrm{NH}_{3}\right)_{2(\mathrm{~s})}+2 \mathrm{NH}_{3(\mathrm{~g})} \rightleftharpoons \mathrm{CaCl}_{2}\left(\mathrm{NH}_{3}\right)_{4(\mathrm{~s})} & \text { III } \\
\mathrm{CaCl}_{2}\left(\mathrm{NH}_{3}\right)_{4(\mathrm{~s})}+4 \mathrm{NH}_{3(\mathrm{~g})} \rightleftharpoons \mathrm{CaCl}_{2}\left(\mathrm{NH}_{3}\right)_{8(\mathrm{~s})} & \text { IV }
\end{array}
$$

Après l'élimination complète de l'ammoniac, on retrouve exactement les caractéristiques cristallographiques du sel pur.

En raison de la faible stabilité relative de l'octo et du tétraammoniacate, ces composés d'addition ont été à la base du principe de fonctionnement d'appareils ménagers permettant de produire du froid [3-6].

Bonauguri [7] semble être le premier à utiliser ces complexes pour la réfrigération solaire en simulant le soleil à l'aide d'une batterie de lampes. Plusieurs problèmes d'ordre technique l'ont contraint à abandonner ses expériences.

\section{Considérations générales.}

Certains auteurs [8] ont observé une baisse de la quantité d'ammoniac désorbée après un certain nombre de cycles de fonctionnement. Mais la majorité s'accorde pour dire que les réactions I à VI restent réversibles [4, 5, 9-11], les ammoniacates gardant leurs propriétés initiales après plusieurs cycles d'absorption suivie de désorption. Normelli $[4,5]$ n'a en effet constaté aucun changement dans le comportement physicochimique des ammoniacates de calcium après leur utilisation durant plusieurs années à raison de 3 cycles par jour.

Pour notre part nous avons expérimenté par simulation rayonnante pendant deux ans ce même couple frigorigène sans observer d'altération notable de la réversibilité des réactions de dissociation et de régénération.

En fait, $\mathrm{CaCl}_{2}$ est un produit très hygroscopique et même déliquescent s'il est exposé suffisamment longtemps à l'air. De plus, le composé $\mathrm{CaCl}_{2}\left(\mathrm{H}_{2} \mathrm{O}\right)_{(\mathrm{s})}$ est très stable et la liaison $\mathrm{CaCl}_{2}-\mathrm{H}_{2} \mathrm{O}$ ne peut être rompue facilement [12]. Pour dessécher complètement $500 \mathrm{~g}$ de $\mathrm{CaCl}_{2}\left(\mathrm{H}_{2} \mathrm{O}\right)_{0,5}$, il a fallu les maintenir à 
$170{ }^{\circ} \mathrm{C}$ en créant un vide primaire durant $48 \mathrm{~h}$. Ces précautions sont essentielles sinon la non-réversibilité des réactions I à IV apparait. Elle peut avoir lieu dans 2 cas :

1) l'appareil dans lequel ont été introduits $\mathrm{CaCl}_{2}$ et $\mathrm{NH}_{3}$ purs n'est pas étanche à l'humidité atmosphérique

2) le chlorure n'est pas complètement déshydraté avant l'absorption d'ammoniac.

Ceci se traduit alors par une décomposition des produits de départ : dans le $1^{\mathrm{er}}$ cas on obtient :

$$
\begin{aligned}
\mathrm{CaCl}_{2}\left(\mathrm{NH}_{3}\right)_{8(\mathrm{~s})} & +\mathrm{H}_{2} \mathrm{O}_{(\mathrm{g})} \rightarrow \\
& \rightarrow \mathrm{CaClOH}_{(\mathrm{s})}+\mathrm{NH}_{4} \mathrm{Cl}_{(\mathrm{s})}+7 \mathrm{NH}_{3(\mathrm{~g})}
\end{aligned}
$$

et dans le second :

$$
\mathrm{CaCl}_{2}\left(\mathrm{H}_{2} \mathrm{O}\right)_{(\mathrm{s})}+\mathrm{NH}_{3(\mathrm{~g})} \rightarrow \mathrm{CaCl}_{2} \mathrm{OH}_{(\mathrm{s})}+\mathrm{NH}_{4} \mathrm{Cl}_{(\mathrm{s})} \text {. }
$$

\section{Gonflement du sel.}

L'absorption chimique de l'absorbat $\left(\mathrm{NH}_{3}\right)$ par l'absorbant $\left(\mathrm{CaCl}_{2}\right)$ entraîne, entre le solide et le gaz, un transfert d'électrons qui se traduit par des liaisons chimiques entre $\mathrm{NH}_{3}$ et la couche externe des atomes de $\mathrm{CaCl}_{2}$. La pénétration du gaz dans la structure du solide se faisant dans la totalité de sa masse par un processus de diffusion. Au cours de cette réaction, la fixation du gaz par le solide s'accompagne d'une augmentation de volume, l'octoammoniacate occupant alors plusieurs fois le volume du sel pur.

Que $\mathrm{CaCl}_{2}$ anhydre soit compact $\left(\rho_{\mathrm{CaCl}_{2}}=\right.$ $2,17 \mathrm{~g} \cdot \mathrm{cm}^{-3}$ ) ou divisé à l'état de grains de quelques millimètres de diamètre, $\mathrm{CaCl}_{2}\left(\mathrm{NH}_{3}\right)_{8(\mathrm{~s})}$ formé est une poudre blanche et fine. Vue sous microscope, elle est constituée de petits grains de différentes formes, leur taille passant de 1 à $25 \mu \mathrm{m}$ environ. L'augmentation de volume du sel est due non seulement à la dilatation de son réseau cristallin mais aussi à son fractionnement laissant ainsi de l'espace libre entre les microcristaux de $\mathrm{CaCl}_{2}\left(\mathrm{NH}_{3}\right)_{8(\mathrm{~s})}$.

En faisant subir à $\mathrm{CaCl}_{2}\left(\mathrm{NH}_{3}\right)_{8(\mathbf{s})}$ une faible pression mécanique de l'ordre de 1 bar, nous avons trouvé une densité de 0,54 $\pm 0,01$. Ce résultat concorde avec ceux existant dans la littérature $[13,14]$.

La figure 1 décrit le gonflement $G$ de l'octoammoniacate en fonction de la densité initiale du sel pur ( $G$ est défini comme le rapport du volume occupé par $\mathrm{CaCl}_{2}\left(\mathrm{NH}_{3}\right)_{8}$ à celui occupé par $\left.\mathrm{CaCl}_{2}\right)$.

En effet, le gonflement observé lors de la fixation de l'ammoniac est fonction de la grosseur des grains de $\mathrm{CaCl}_{2}$ initial. Aussi avons-nous utilisé différents tamis afin de procéder à une sélection granulométrique, la masse volumique du sel augmentant lorsque la taille des grains diminue.

A partir de ces différents résultats, nous déduisons que dans la conception de l'absorbeur (ou générateur), il faut prévoir un volume libre de 41 environ pour $1 \mathrm{~kg} \mathrm{de} \mathrm{CaCl}_{2}$ pur. Par contre, si le volume disponible

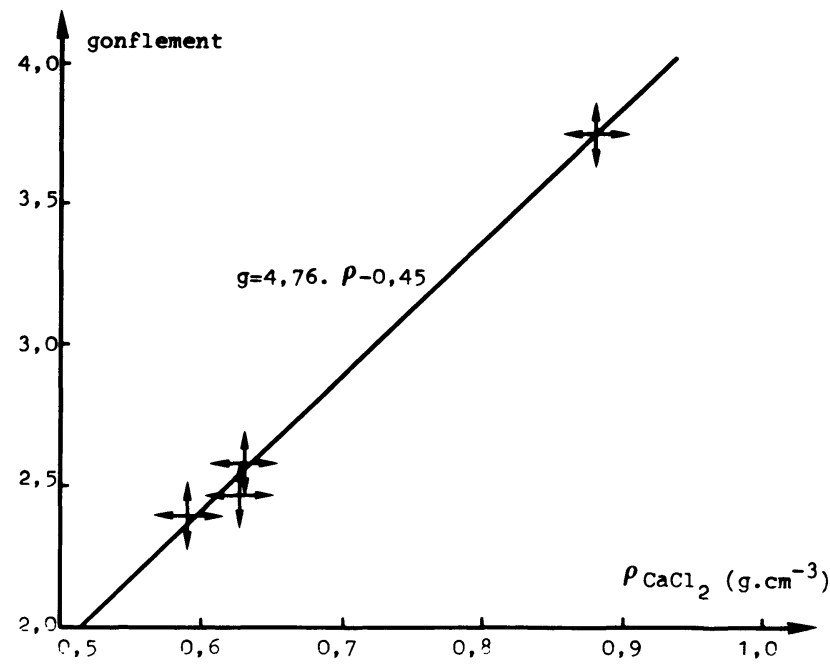

Fig. 1. - Gonflement de l'octoammoniacate en fonction de la masse volumique du sel pur.

[Swelling of the octoammoniacate in function of the pure salt volumic mass.]

est trop petit, l'absorption de $\mathrm{NH}_{3}$ risque de s'interrompre.

En définitive, le gonflement considérable du sel n'est pas nécessairement un désavantage puisque l'état divisé de l'ammoniacate améliore les vitesses d'absorption et de désorption et diminue les gradients de pression dans la masse et par conséquent les gradients de concentration. L'étude par diffraction $\mathbf{X}$ de $\mathrm{CaCl}_{2}\left(\mathrm{NH}_{3}\right)_{8(\mathbf{s})}$ (Westman et al. [15]) conduit à la conclusion que le volume occupé dans le réseau cristallin par une molécule d'octoammoniacate est 4 fois plus grand que celui occupé par une molécule de $\mathrm{CaCl}_{2}$. Du point de vue macroscopique, lorsque le gonflement est optimal, $\mathrm{CaCl}_{2}\left(\mathrm{NH}_{3}\right)_{8}$ occupe un volume partiellement lacunaire, les vides résultant de l'effritement du complexe.

\section{Cinétiques d'absorption et de désorption de $\mathbf{N H}_{3}$.}

4.1 APPAREILlage. - La réfrigération solaire par absorption sèche est limitée dans le temps à un cycle par $24 \mathrm{~h}$. Il est donc important d'évaluer les temps nécessaires à la décomposition et à la synthèse des composés solides. Pour étudier ce problème, nous avons construit un petit appareil constitué de 2 enceintes en acier communiquant entre elles :

\section{- un évaporateur condenseur}

- un absorbeur-générateur.

Le condenseur (C) est un tube cylindrique horizontal ayant un volume de 11 . Il est entouré d'un serpentin où peut circuler de l'eau le maintenant à température constante. Une graduation diamétrale du tube permet de suivre la quantité présente d'ammoniac liquide à travers 2 plaques de verre de $1 \mathrm{~cm}$ d'épaisseur à l'épreuve de 25 bars et se trouvant aux deux extrémités du cylindre, protégées par 2 plaques d'acier convenablement évidées afin de permettre les lectures. 


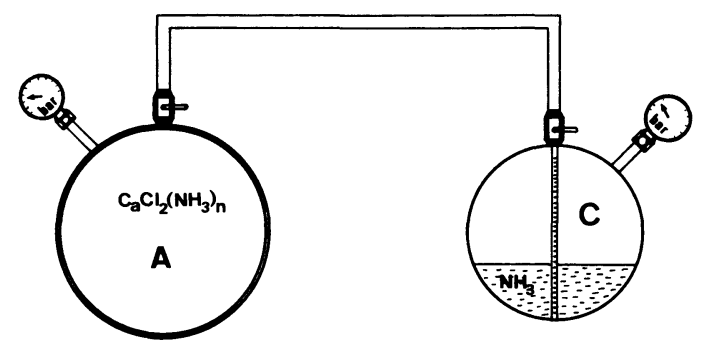

- Schéma de l'appareil.

[Schema of the apparatus.]

Un thermocouple introduit au fond du tube à travers un passage étanche indique la température du fluide tandis qu'un manomètre "Bourdon » donne sa pression.

Le générateur (A) qui contient le sel saturé d'ammoniac est un tube cylindrique également horizontal de volume 21 . Il est entouré d'une résistance électrique chauffante permettant d'atteindre $200^{\circ} \mathrm{C}$. Trois thermo-couples introduits à l'aide de passages étanches en des points d'abscisses et de cotes différentes à partir de l'axe du tube précisent la température du sel. Deux manomètres "Bourdon" gradués l'un de 0 à 1 bar, l'autre de 1 à 26 bars mesurent la pression à l'intérieur de l'enceinte. Comme pour le condenseur, 2 plaques de verre épais permettent de suivre l'évolution macroscopique du sel durant l'absorption et la désorption.
En tenant compte du gonflement résultant de l'absorption de $\mathrm{NH}_{3}$ afin que le sel ne subisse pas de contraintes excessives de la part des parois du générateur, nous avons introduit $550 \mathrm{~g} \mathrm{de} \mathrm{CaCl}_{2}$ que nous avons desséché en le chauffant à $170^{\circ} \mathrm{C}$ sous vide primaire. Un piège à air liquide a permis de recueillir $14 \mathrm{~g}$ d'eau saumâtre après $48 \mathrm{~h}$ de traitement. Le $3^{\mathrm{e}}$ jour, la quantité d'eau exsudée était négligeable.

4.2 VITESSES D'ABSORPTION OU FORMATION DES AMMONIACATES. - A partir de $\mathrm{CaCl}_{2}$ anhydre, nous avons formé $\mathrm{CaCl}_{2}\left(\mathrm{NH}_{3}\right)_{8}$ en 8 étapes, chacune consistant à obtenir l'ammoniacate $\mathrm{CaCl}_{2}\left(\mathrm{NH}_{3}\right)_{n+1}$ à partir de $\mathrm{CaCl}_{2}\left(\mathrm{NH}_{3}\right)_{n}$.

Connaissant le niveau de $\mathrm{NH}_{3}$ liquide dans le condenseur, sa température, la pression du gaz dans le condenseur et le générateur, la température du sel et le volume disponible pour le gaz dans l'appareil, nous en déduisons la masse d'ammoniac absorbée par le sel à chaque instant et par conséquent le nombre $N$ de moles de $\mathrm{NH}_{3}$ absorbées par mole de $\mathrm{CaCl}_{2}$.

Nous diviserons en 2 groupes l'étude de ces étapes.

4.2.1 Formation des ammoniacates 1 et 2 . - La figure 2 montre la variation de $N$ en fonction du temps pour les 2 réactions :

$$
\begin{array}{ll}
\mathrm{CaCl}_{2(\mathrm{~s})}+\mathrm{NH}_{3(\mathrm{~g})} & \rightarrow \mathrm{CaCl}_{2}\left(\mathrm{NH}_{3}\right)_{(\mathrm{s})} \\
\mathrm{CaCl}_{2}\left(\mathrm{NH}_{3}\right)_{(\mathrm{s})}+\mathrm{NH}_{3(\mathrm{~g})} & \rightarrow \mathrm{CaCl}_{2}\left(\mathrm{NH}_{3}\right)_{2(\mathrm{~s})} .
\end{array}
$$

Durant l'absorption, nous avons observé une sub-

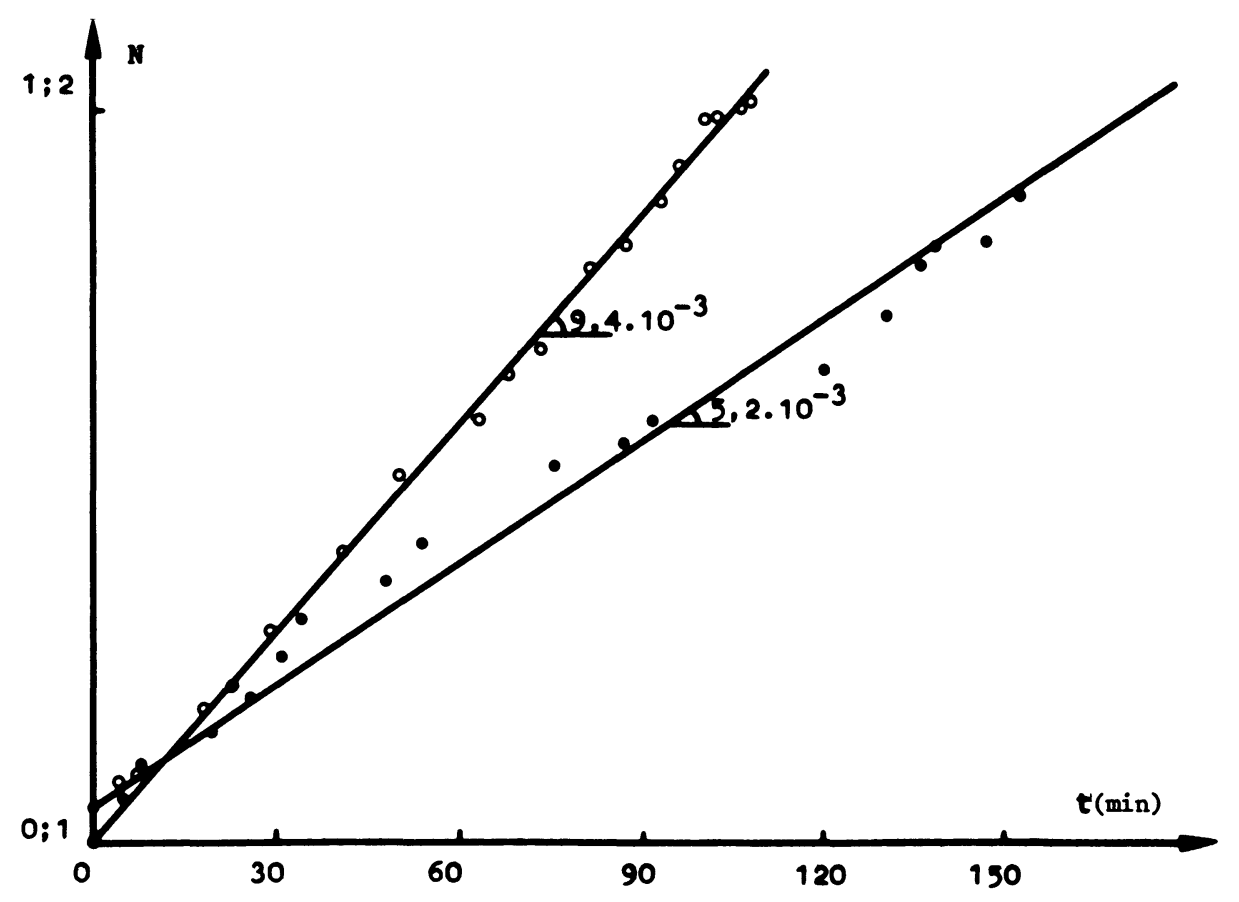

Fig. 2. - Variation en fonction du temps, du nombre $N$ de moles de $\mathrm{NH}_{3}$ absorbées par mole de $\mathrm{CaCl}_{2}$ à pression constante $N: 0 \rightarrow 1 \circ N: 1 \rightarrow 2$.

[Variation in function of the time of $\mathrm{NH}_{3}$ moles number, absorbed by one mole of $\mathrm{CaCl}_{2}$ at constant pressure.] 
division des grains de sel correspondant à un processus de germination favorisant ainsi le processus réactionnel.

Dans le cas de ces 2 réactions, nous avons maintenu à 1 bar la pression du gaz dans l'absorbeur. A cette pression, la masse d'ammoniac $\Delta M$ absorbée par le sel est une fonction linéaire du temps puisque $N$ est reliée à $\Delta M$ par :

$$
N(t)=\alpha+\beta . \Delta M(t) \quad \alpha \text { et } \beta \text { constantes } .
$$

Dans les mêmes conditions expérimentales, $\mathrm{CaCl}_{2}\left(\mathrm{NH}_{3}\right)_{(\mathrm{s})}$ s'est formé 1,8 fois plus vite que $\mathrm{CaCl}_{2}\left(\mathrm{NH}_{3}\right)_{2}$.

4.2.2 Formation des ammoniacates 3 à 8. - Ici, nous avons fait circuler l'eau dans le serpentin pour maintenir le condenseur à température constante. Une forte différence de pression (6 à 7 bars) existait entre les 2 enceintes avant de les mettre en communication de sorte qu'au début, l'absorption est tellement forte que la température du condenseur baisse rapidement par suite de l'évaporation importante de l'ammoniac liquide. Cette opération dure entre 4 et $8 \mathrm{~min}$, le système n'étant pas en équilibre. La circulation d'eau permet au système de retrouver un certain équilibre et la pression du gaz se stabilise pour ne varier que de quelques dixièmes de bar jusqu'à la disparition complète de l'ammoniac liquide. A partir de ce moment, l'absorption devient très lente, la pression du gaz diminuant progressivement.

Nous subdiviserons donc chaque réaction en 3 parties :

- la $1^{\text {re }}$ est un état de déséquilibre sans grand intérêt ;
- la $2^{\mathrm{e}}$ est un état de quasi-équilibre. La pression dans les deux enceintes ne variant que très peu, nous pourrions donc retrouver des droites $N=a t+b$, $a$ et $b$ constantes. C'est ce que montre la figure 3 où les droites représentées résultent d'un alignement linéaire par la méthode des moindres carrés ;

- la $3^{\mathrm{e}}$ est sans importance pratique puisque la quantité de $\mathrm{NH}_{3}$ absorbée est très faible, $N$ n'augmentant au maximum que de 0,06 mole de $\mathrm{NH}_{3}$ par mole de $\mathrm{CaCl}_{2}$.

Le tableau I donne la valeur des pentes des droites durant la formation des divers ammoniacates dans les conditions expérimentales retenues ainsi que les pentes réduites à 1 bar de $\mathrm{CaCl}_{2}\left(\mathrm{NH}_{3}\right)_{3}$ à $\mathrm{CaCl}_{2}\left(\mathrm{NH}_{3}\right)_{8}$ en supposant que la vitesse de formation des ammoniacates soit proportionnelle à la pression du gaz.

Nous observons sur ce tableau qu'à pression constante, la formation de $\mathrm{CaCl}_{2}\left(\mathrm{NH}_{3}\right)_{8}$ (à partir de $\left.\mathrm{CaCl}_{2}\left(\mathrm{NH}_{3}\right)_{7}\right)$ est 4 fois moins rapide que celle de $\mathrm{CaCl}_{2}\left(\mathrm{NH}_{3}\right)_{5}$ (à partir de $\left.\mathrm{CaCl}_{2}\left(\mathrm{NH}_{3}\right)_{4}\right)$ et 2 fois plus faible que celle de $\mathrm{CaCl}_{2}\left(\mathrm{NH}_{3}\right)_{\mathrm{E}}$.

Afin de nous rapprocher des conditions pratiques d'utilisation du réfrigérateur solaire, nous avons examiné 2 expériences différentes durant la phase d'évaporation de l'ammoniac. Les résultats sont portés sur la figure 4 : la courbe 1 est relative à la formation de l'octoammoniacate à partir de $\mathrm{CaCl}_{2}\left(\mathrm{NH}_{3}\right)_{2,5}$ ? A. l'instant $t=0$, la température du sel était de $70^{\circ} \mathrm{C}$ et l'évaporateur était à $9^{\circ} \mathrm{C}$. L'absorption s'est faite de façon continue, les pressions étant les mêmes dans les 2 enceintes et la température de l'évaporateur décroissait au fur et à mesure que se produisait l'évaporation. Cette variation de $N$ correspond à celle qu'on obtiendrait au niveau de l'absorbeur d'un réfrigérateur solaire et en fin d'après-midi.

Tableau I. - Vitesse de formation des 8 ammoniacates.

\begin{tabular}{|c|c|c|c|}
\hline Réaction & $\begin{array}{c}\text { Pression } \\
\text { (bar) }\end{array}$ & $\begin{array}{c}\text { Pente } \\
(\text { mole.min }\end{array}$ & $\begin{array}{c}\text { Pente réduite à } \\
1 \text { bar } \\
\left(\text { mole.min }^{-1}\right)\end{array}$ \\
\hline $\mathrm{CaCl}_{2} \rightarrow \mathrm{CaCl}_{2}\left(\mathrm{NH}_{3}\right)$ & 1,0 & $9,4 \times 10^{-3}$ & $9,4 \times 10^{-3}$ \\
\hline $\mathrm{CaCl}_{2}\left(\mathrm{NH}_{3}\right) \rightarrow \mathrm{CaCl}_{2}\left(\mathrm{NH}_{3}\right)_{2}$ & 1,0 & $5,2 \times 10^{-3}$ & $5,2 \times 10^{-3}$ \\
\hline $\mathrm{CaCl}_{2}\left(\mathrm{NH}_{3}\right)_{2} \rightarrow \mathrm{CaCl}_{2}\left(\mathrm{NH}_{3}\right)_{3}$ & 5,5 & $2,4 \times 10^{-2}$ & $4,3 \times 10^{-3}$ \\
\hline $\mathrm{CaCl}_{2}\left(\mathrm{NH}_{3}\right)_{3} \rightarrow \mathrm{CaCl}_{2}\left(\mathrm{NH}_{3}\right)_{4}$ & 5,6 & $1,8 \times 10^{-2}$ & $3,2 \times 10^{-3}$ \\
\hline $\mathrm{CaCl}_{2}\left(\mathrm{NH}_{3}\right)_{4} \rightarrow \mathrm{CaCl}_{2}\left(\mathrm{NH}_{3}\right)_{5}$ & 5,7 & $1,8 \times 10^{-2}$ & $3,1 \times 10^{-3}$ \\
\hline $\mathrm{CaCl}_{2}\left(\mathrm{NH}_{3}\right)_{5} \rightarrow \mathrm{CaCl}_{2}\left(\mathrm{NH}_{3}\right)_{6}$ & 5,9 & $1,5 \times 10^{-2}$ & $2,5 \times 10^{-3}$ \\
\hline $\mathrm{CaCl}_{2}\left(\mathrm{NH}_{3}\right)_{6} \rightarrow \mathrm{CaCl}_{2}\left(\mathrm{NH}_{3}\right)_{7}$ & 5,8 & $1,0 \times 10^{-2}$ & $1,7 \times 10^{-3}$ \\
\hline $\mathrm{CaCl}_{2}\left(\mathrm{NH}_{3}\right)_{7} \rightarrow \mathrm{CaCl}_{2}\left(\mathrm{NH}_{3}\right)_{8}$ & 6,0 & $5,0 \times 10^{-2}$ & $0,83 \times 10^{-3}$ \\
\hline
\end{tabular}




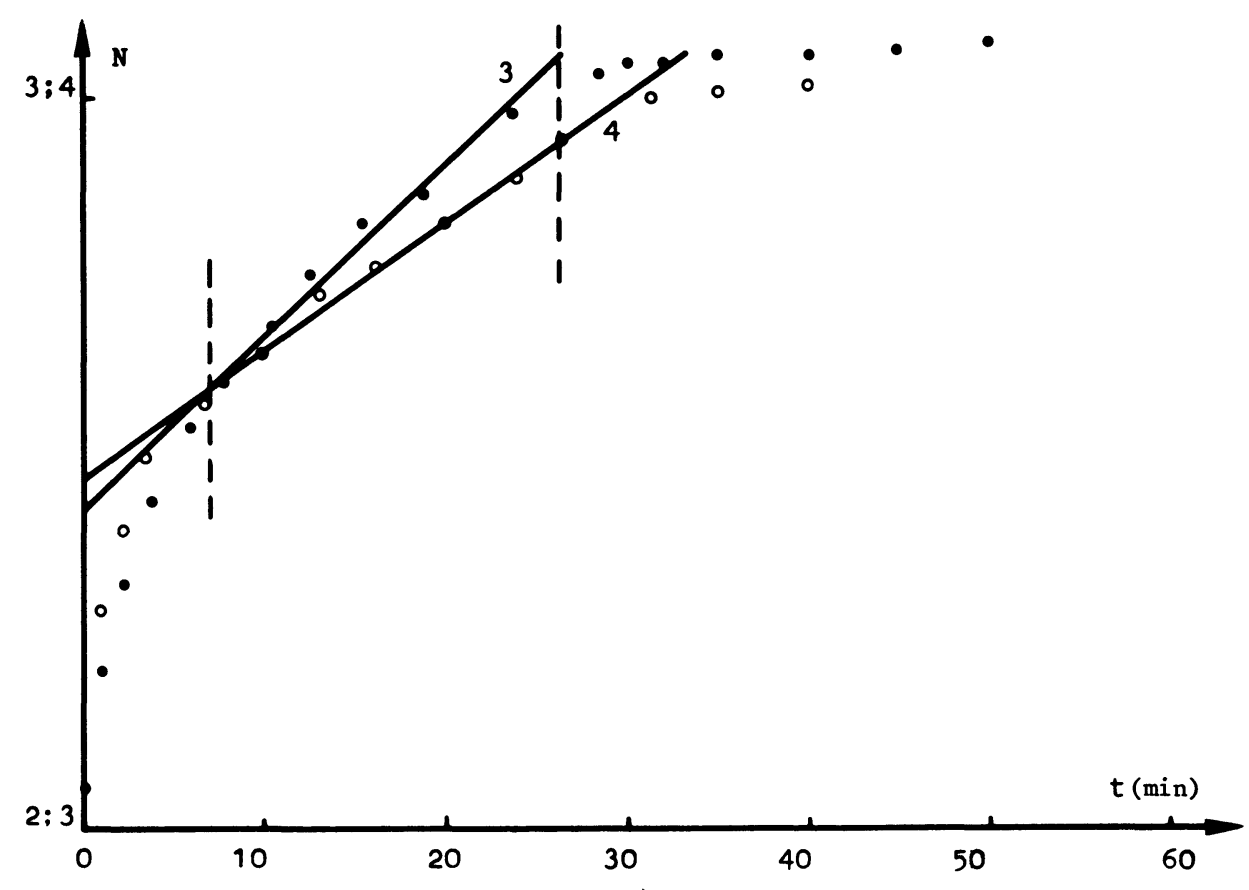

a)

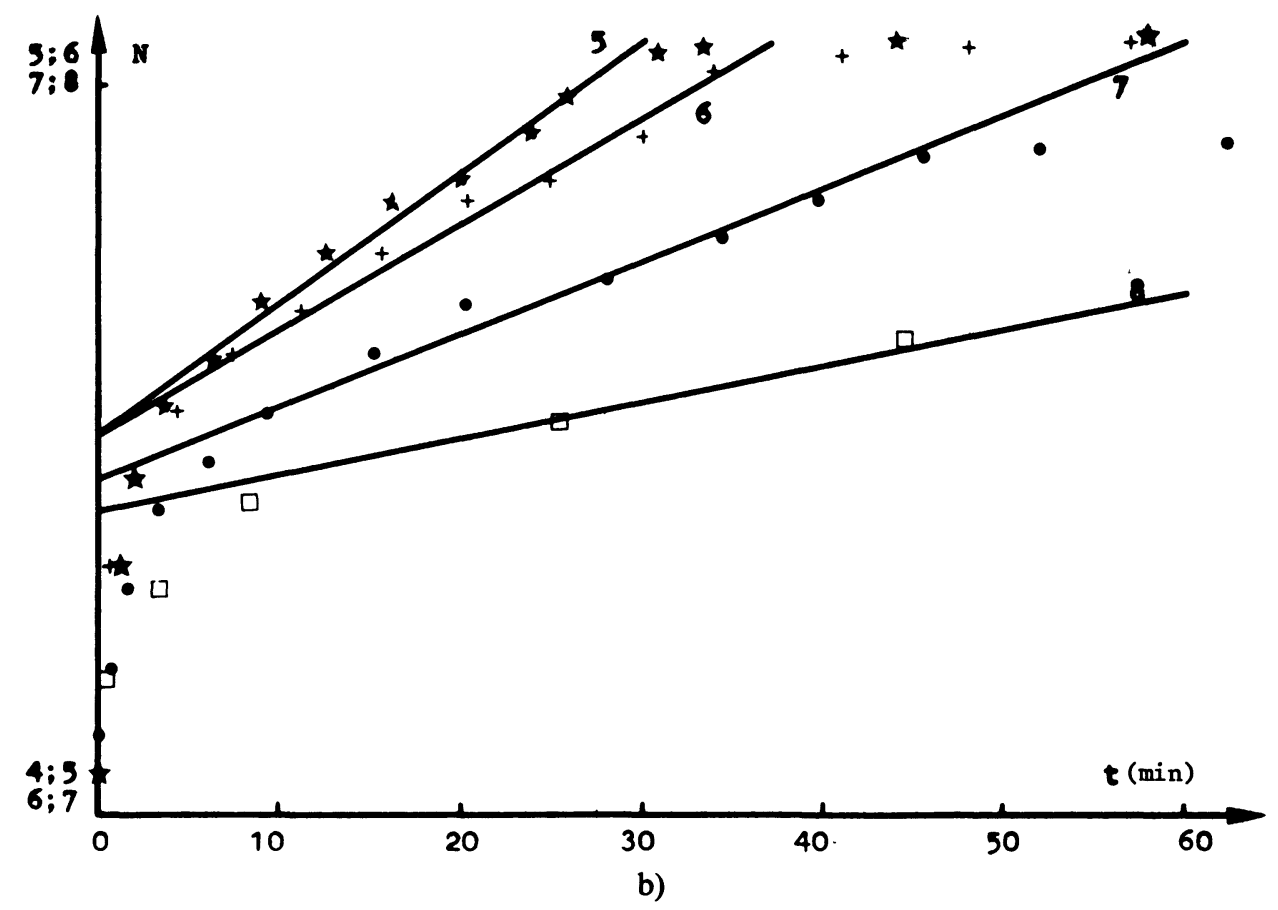

Fig. 3. - a) Variation de $N$ 3-4. b) Variation de $N$ 5-8.

[Variation of $N$ 3-4. Variation of $N$ 5-8.]

La loi empirique $N(t)=2,5+1,1 \times 10^{-2} t-$ $8,8 \times 10^{-6} t^{2}+4,6 \times 10^{-4} t$. Ln $t$ décrit convenablement les valeurs obtenues pour $N$.

La courbe 2 montre la variation de $N$ entre 4 et 8 . Une loi de même forme que la précédente décrit cette variation :

$N(t)=4,0+4,3 \times 10^{-2} t+9,8 \times 10^{-6} t^{2}-$ $-6,3 \times 10^{-3} t . \operatorname{Ln} t$.
L'écart de $N$ à cette loi en début d'expérience vient du fait que la température du sel au départ était de $30^{\circ} \mathrm{C}$, le sel absorbant l'ammoniac d'autant plus vite que sa température est basse.

En définitive et dans un petit réacteur, l'absorption d'ammoniac au cours d'une nuit est compatible avec un cycle de $24 \mathrm{~h}$ puisque $\mathrm{CaCl}_{2}\left(\mathrm{NH}_{3}\right)_{8}$ se formera en $10 \mathrm{~h}$ environ à condition de réserver à l'ammoniacate un espace libre suffisant dans l'absorbeur. 


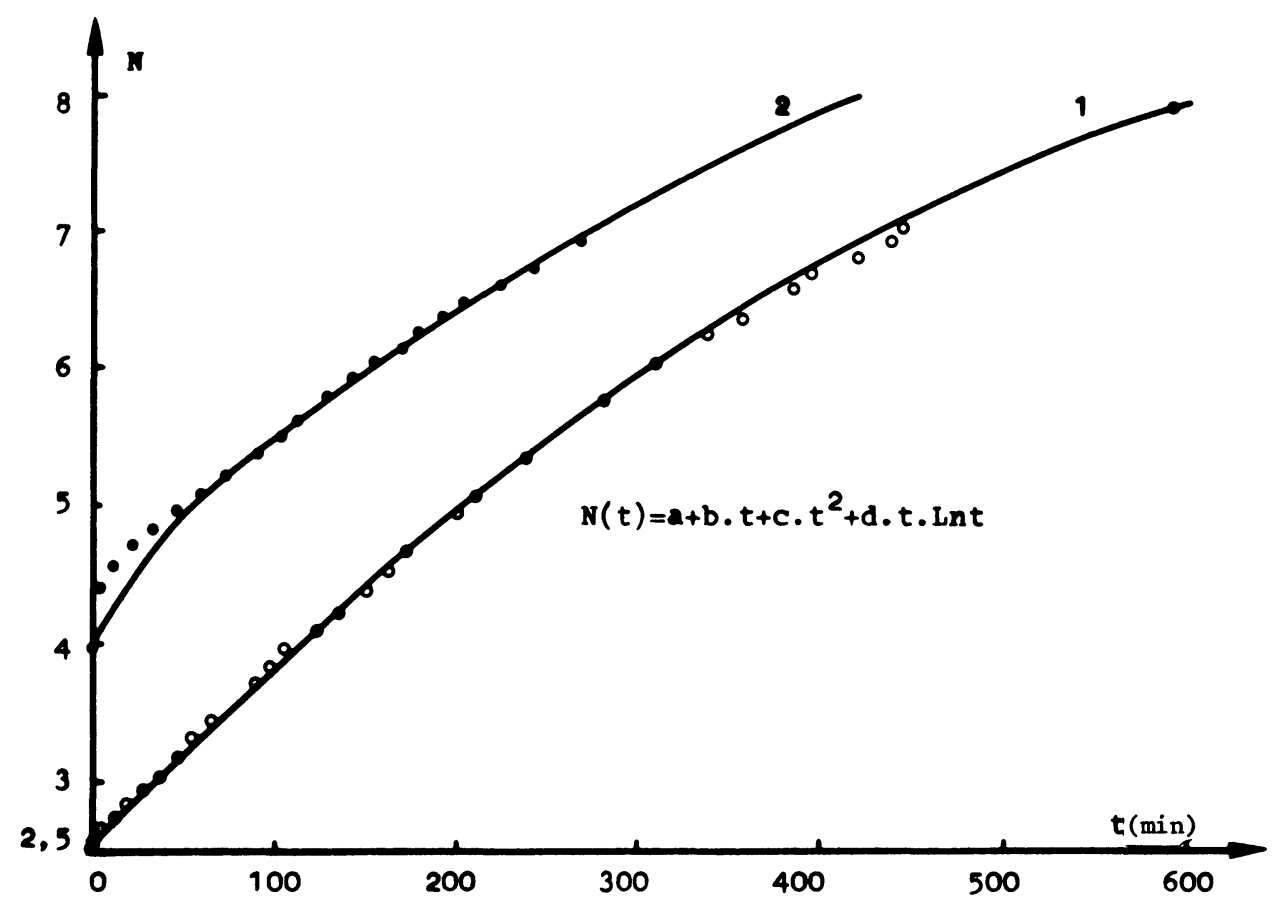

Fig. 4. - Cinétique de formation des ammoniacates.

[Ammoniacates formation kinetics.]

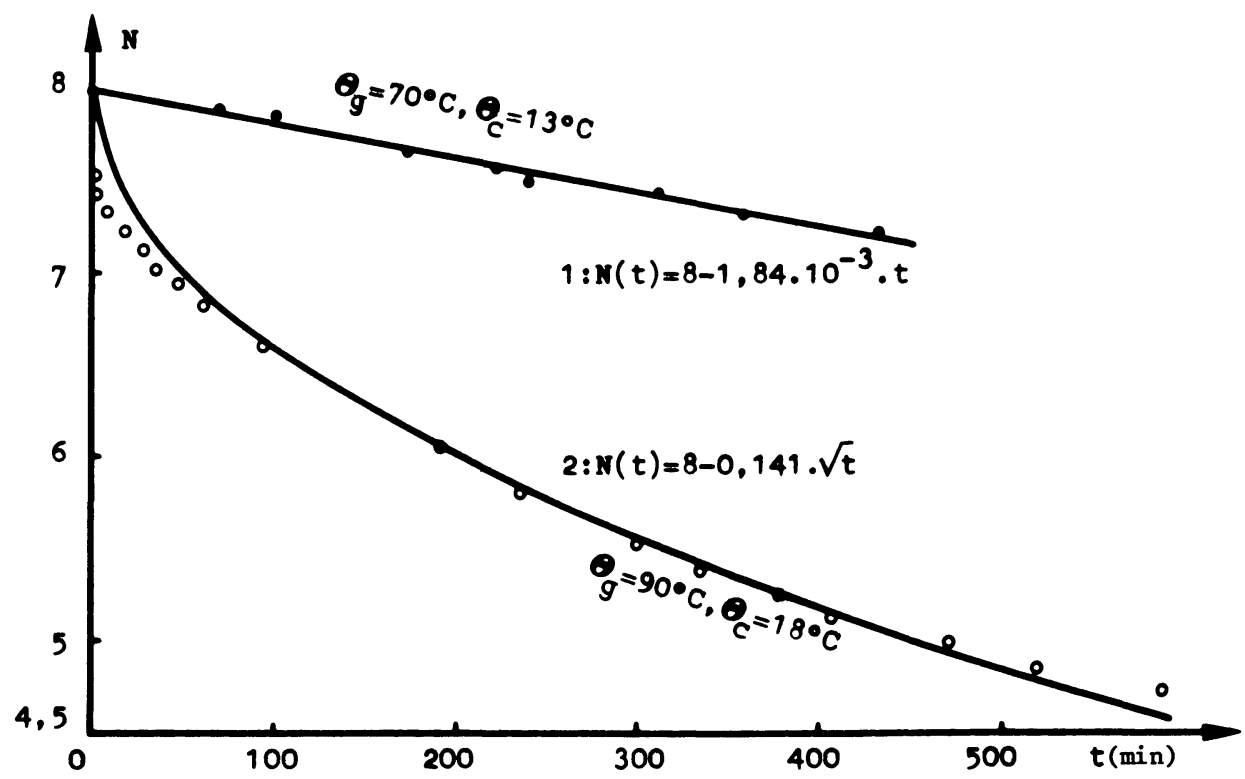

Fig. 5. - Vitesse de désorption de l'ammoniac à température constante.

[Desorption rate of ammonia at constant temperature.]

4.3 VITESSES DE DÉCOMPOSITION DE L'OCTOAMMONIACATE. - Nous avons examiné l'effet de la température du sel sur sa vitesse de dissociation. Sur la figure 5, la courbe 1 décrit cette dissociation lorsque le sel est maintenu constamment à $70^{\circ} \mathrm{C}$ et le condenseur à $13^{\circ} \mathrm{C}$. La variation de $N$ est linéaire et suit la loi $N(t)=8 \times 1,84 \times 10^{-3} . t$. Par contre, lorsque le sel est maintenu à $90^{\circ} \mathrm{C}$ et le condenseur à $18^{\circ} \mathrm{C}$ (courbe 2), cette variation n'est plus linéaire mais suit la loi :

$$
N(t)=8-0,141 \sqrt{t} .
$$

Une très forte différence apparaît puisque dans le $1^{\text {er }}$ cas (courbe 1) à peine 0,8 mole de $\mathrm{NH}_{3}$ est libérée 
en $7 \mathrm{~h}$ alors qu'un sel à $90^{\circ} \mathrm{C}$ en rejette 3 dans le même temps bien que le condenseur soit à une température plus élevée.

Dans le but de déterminer les performances d'une expérience réelle, nous procédons par simulation. En effet, à l'aide d'un rhéostat branché sur la résistance électrique enveloppant le générateur, nous avons simulé la montée en température du sel à partir d'un enregistrement réalisé au soleil de Nancy, par ciel clair, sur un capteur cylindro-parabolique, le tube focal contenant de l'huile. La température du condenseur était maintenue à l'aide d'une autre résistance électrique à $40^{\circ} \mathrm{C}$ avant le début de la condensation de l'ammoniac puis à $50^{\circ} \mathrm{C}$ durant cette période. La figure 6 montre les résultats de cette expérience qui correspondrait à la dissociation de l'octoammoniacate en milieu torride.

Nous pouvons décrire qualitativement la variation de $N$ par des droites correspondant à 3 régions différentes :

a) 1 re région : le condenseur étant sensiblement à $40^{\circ} \mathrm{C}$ et la température $\theta_{\mathrm{g}}$ du sel variant de 40 à $87,5^{\circ} \mathrm{C}, N$ décroît très lentement pour ne varier que de 0,15 mole $\mathrm{NH}_{3} / \mathrm{mole} \mathrm{CaCl}_{2}$. Cette période qui dure $2,5 \mathrm{~h}$ est terminée quand la pression du gaz dans le condenseur devient saturante (ici $P_{\mathrm{s}}=15,5$ bars). Elle est d'autant plus courte que la température du condenseur est moins élevée pour la même température du sel. b) $2^{\mathrm{e}}$ région : c'est la période où la variation de $N$ est la plus forte. Elle débute dès que des gouttelettes d'ammoniac apparaissent sur la paroi interne du condenseur et s'arrête dès que le sel atteint sa température maximale en cours de journée (entre $11 \mathrm{~h}$ et 13 h T.S.V.). Pour 2,5 h, $N$ varie de 3,17 moles $\mathrm{NH}_{3} /$ mole $\mathrm{CaCl}_{2}$. La pression de l'ammoniac se maintenait aux environs de 20 bars pour une température au condenseur de $50^{\circ} \mathrm{C}$.

c) $3^{\mathrm{e}}$ région : lorsque la température du sel atteint son maximum (entre $11 \mathrm{~h}$ et $13 \mathrm{~h}$ T.S.V.) pour décroître ensuite, la variation est moins marquée que précédemment. Pourtant, durant cette période qui dure un peu moins de $3 \mathrm{~h}, N$ décroît encore de $1,2 \mathrm{~mole} \mathrm{NH}_{3} / \mathrm{mole}$ $\mathrm{CaCl}_{2}$. Au-delà, et bien que la température du sel demeure élevée (entre $130^{\circ} \mathrm{C}$ et $80^{\circ} \mathrm{C}$ ), la dissociation se bloque complètement : il n'y a plus d'apport d'ammoniac au condenseur.

A la fin de cette expérience, le composé restant dans le générateur est $\mathrm{CaCl}_{2}\left(\mathrm{NH}_{3}\right)_{3,5}$, ceci pour une période d'ensoleillement active de $8 \mathrm{~h}$. La concentration massique en ammoniac est donc passée de $55,1 \%$ à $34,7 \%$. Cela signifie que si l'on disposait initialement de $10 \mathrm{~kg}$ de $\mathrm{CaCl}_{2}$ pur saturé en ammoniac, dans les mêmes conditions expérimentales, on distillerait 12,251 d'ammoniac liquide.

Un recyclage du système conduit à des résultats analogues.

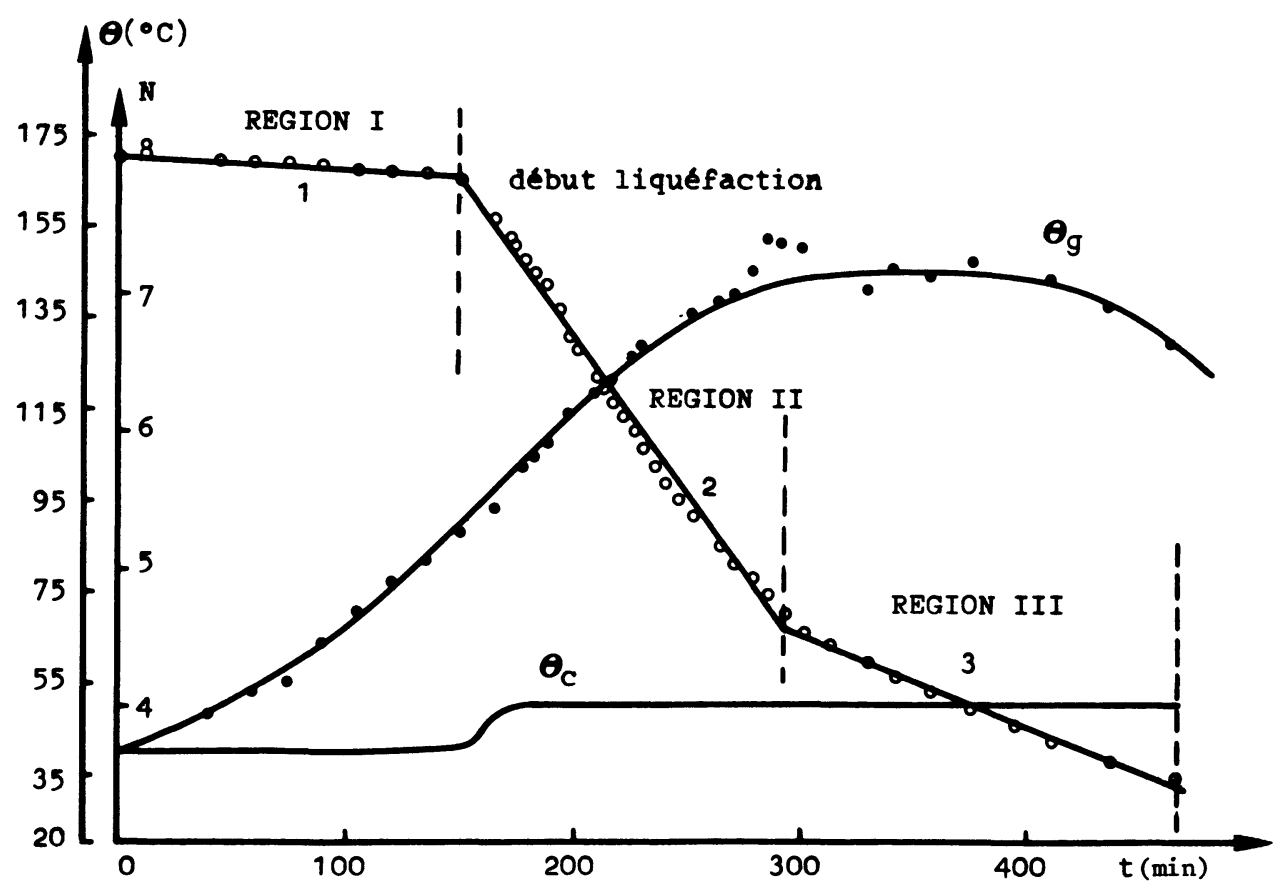

Fig. 6. - Simulation des effets du soleil sur le gel pendant la désorption d'ammoniac. $\theta_{\mathbf{g}}$ Température du générateur $\theta_{\mathbf{c}}$ Température du condenseur.

[Simulation of the solar effects on the salt during the ammonia desorption.] 


\section{Conclusion.}

Conformément au but que nous nous sommes proposé, cette étude par simulation appuyée sur des résultats obtenus sous un ensoleillement réel, nous permet de définir les possibilités et les limites de cette méthode photothermique appelée à répondre aux besoins de conservation des denrées périssables dans une ambiance tropicale caractérisant de nombreux pays en voie de développement à l'intention desquels l'ensemble de ce travail a été entrepris.
Ce texte est le second d'une série qui s'achèvera par un examen énergétique de phénomènes observés au cours de cette expérimentation.

$N B$ : En juillet 1984 une demande de brevet a été déposée par l'ANVAR sur un appareil industrialisé issu de ces recherches (surface captante de $7 \mathrm{~m}^{2}$, cuve isolante de 300 1).

Un prototype est actuellement installé pour tests in situ à Madagascar. Payé par l'UNESCO, un second prototype a été monté en septembre 1985 au Centre International de Physique Théorique de Triestre.

\section{Bibliographie}

[1] Hüttig, Z., Anorg. Chem., 123, 31 (1922).

[2] Hart, A. B., Partington, J. R., " Dissociation pressures of compounds of ammonia and tridenterammonia with some metallic salts ", J. Chem. Soc., 104 (1943).

[3] LINGE, K., "Uber periodische absorptionk ältemaschine ", D. Sc. Thesis, Karlsruhe (1929).

[4] Normelli, W. B., « Periodische Absorptionkältemaschine ", D.R.P. 554766 (1932).

[5] Normelli, W. B., « Periodische Absorptionskältemaschine ", D.R.P. 549343 (1932).

[6] Planck, R., KuPrianoff, J., « Die Kleinkältemaschine ", Spring Verlag, Berlin (1960).

[7] Bonauguri, E., « Preliminary notes on a solar absorption unit ", Proc. 12th Int. Cong. Ref. Madrid, Vol. 2 (1967).

[8] Wentworth, W. E., Johnson, D. W., Raldow, W. M., "Chemical heat pump using a dispersion of a metal salt ammoniate in an inert solvant ", Solar Energy, 26 (1981) 141-146.

[9] ANDREWS, H. I., "The development of a solid-absorption refrigerating machine for use on trains", Modern Refrigeration, 58 (1955) 330-336.

[10] JAEGER, F. A., "Thermochemical energy storage systems", Proc. 2nd Annual Thermal Energy Storage, Gatlinburg (Tennessee) (1977) 325-332.

[11] Howerton, M. T., « A thermochemical energy storage system and heat pumps ", Proc. Intersoc. Energy Conversion, 13(2), (1978) 935-940.

[12] Fléchon, J., Machizaud, F., Benhammou, K., GodMEL, G., " Réfrigération solaire par photothermie. Premiers résultats d'un appareil réel», Revue Phys. Appl. 19 (1984) 601.

[13] Wentworth, W. E., "Chemical heat pump reactions using metal salt ammoniac complexes in suspensions ", International Seminar on Thermochemical Energy Storage, Stockholm (1980) 371-397.

[14] Mauran, S., Bodiot, D., Crosat, G., "Optimisation des densités énergétiques des systèmes de stockage chimique basés sur des réactions solide-gaz renversables ", Revue Phys. Appl. 18 (1983) 107-112.

[15] Westman, S., Werner, P. E., Schuller, T., RalDOW, W., "X-Ray investigations of ammines of alkaline earth metal halides. I The structure of $\mathrm{CaCl}_{2}\left(\mathrm{NH}_{3}\right)_{8}, \mathrm{CaCl}_{2}\left(\mathrm{NH}_{3}\right)_{2}$ and the decomposition product $\mathrm{CaClOH} »$, Acta Chemica Scandinavia A 35 (1981) 467-472. 